
April 29 - May 2, 2019, Leuven, Belgium

\title{
Bicyclist Route Choice: Data Exploration and Research Project Outline
}

\author{
Luk Knapen ${ }^{\mathrm{a}, \mathrm{b}, *}$, Thomas Koch ${ }^{\mathrm{c}}$, Elenna Dugundji ${ }^{\mathrm{a}, \mathrm{c}}$ \\ ${ }^{a}$ VU University, De Boelelaan 1081a, 1081 HV Amsterdam, The Netherlands \\ ${ }^{b}$ UHASSELT, Agoralaan - Gebouw D, 3590 Diepenbeek, Belgium \\ ${ }^{c}$ Centrum Wiskunde E Informatica, Science Park 123, 1098 XG Amsterdam, The Netherlands
}

\begin{abstract}
Microsimulation of travel flows aims to assess the effect of decisions taken by travelers based on personal preferences, time-ofday, properties of the infrastructure and expected or perceived travel flows. Route choice represents a particular class of such decisions. Route choice prediction is an essential component of microsimulators. Specification of choice models and estimation of the corresponding parameters based on observations are required in the preparatory stage. Route choice sets need to be established for sampling in the simulation stage.

This paper is part of a research project aiming to investigate how route complexity can be integrated in the choice process modeling. In particular routes for bikers collected by GPS tracking in the Dutch FietsTelWeek project in 2016 are analyzed.

The data exploration stage and the research project outline are covered. Properties of the publicly available fietstelweek2016 dataset used for model training are investigated in order to assess their effect on prediction results. In order to achieve the project goal, the research project structure is briefly discussed. It is based on the observation that the number of routes recorded for each OD-pair is too small to observe a frequency distribution for complexity. Hence, complexity data are collected for sub-networks that are similar with respect to particular graph properties.
\end{abstract}

(C) 2019 The Authors. Published by Elsevier B.V.

This is an open access article under the CC BY-NC-ND license (http://creativecommons.org/licenses/by-nc-nd/4.0/) Peer-review under responsibility of the Conference Program Chairs.

Keywords: GPS traces; bicycle; route; choice set

\begin{tabular}{|llll|}
\hline \multicolumn{3}{c|}{ Nomenclature } \\
\hline BFS-LE & Breadth First Search - Link Elimination & BPC & Basic Path Component \\
CDF & Cumulative Distribution Function & CSN & Consideration SubNetwork \\
DSCSG & Doubly Stochastic Choice Set Generation & LCP & Limited Complexity Paths set \\
MNL & Multinomial Logit & PWF & Probability Weight Function \\
POSDAP & Position Data Processing (ETHZ) & PSL & Path Size Logit \\
RL+LS & Recursive Logit + Link Size & SPT & Shortest Path Tree \\
\hline
\end{tabular}

\footnotetext{
* Corresponding author. Tel.: +32-11-26.91.11; fax: +32-11-26.91.99

E-mail address: luk.knapen@uhasselt.be
} 


\section{Research Context and Problem Statement}

Route choice sets are required for two purposes: (i) for parameter estimation in route based choice models [2] and (ii) for route sampling in microsimulation in order to apply travel demand to the road network. The use of a choice set for model estimation can be avoided by applying a recursive logit model. However, in order to apply route choice models in stochastic travel simulators, candidate routes need to be generated and compared. Discrete choice models are used to predict the probability for a route in the choice set from socio-demographic properties of the individual, the trip purpose and the actual network state. In this study, path complexity is introduced as an additional route attribute along with the classical ones (distance, travel time, number of left turns, road type, scenery, ...).

The complexity of a given (observed) path in a graph is related to the minimum number of least-cost paths that is required to specify that path in the network. It can be interpreted as the (minimum) number of intermediate destinations the traveler may have in mind and that are connected by least-cost subpaths. Note that complexity is a graph theoretical property and is not related to geometric properties of the route. Complexity is a path attribute which is considered to be important for route choice.

It has been observed by [7] that the distributions for the path complexity for sets of observed routes on one hand and predicted (by the POSDAP DSCSG method) routes on the other hand may drastically differ. Ongoing research shows that the distribution for the path complexity for routes predicted by the POSDAP BFS-LE method also heavily deviates from the observed one. DSCSG seems to heavily overestimate complexity whereas BFS-LE seems to heavily underestimate complexity.

In this study we aim to create choice sets for micro-simulation having a probability weight function (PWF) for path complexity that conforms to the observed reality. Hence, we propose to use complexity in the selection criteria when populating choice sets for microsimulation.

Hereafter, probability weight functions (PWF) and the corresponding cumulative distribution functions (CDF) for route complexity will be denoted by $f_{c}$ and $F_{c}$ respectively. Additional subscripts and superscripts are used to distinguish particular path sets.

\section{State of the Art}

Due to lack of space, the literature review is limited and focuses on recursive logit models and route complexity because they constitute the basis of the proposed research. Route based choice models that require a choice set for model estimation are not discussed.

\subsection{Route Choice Modeling}

Recursive logit (RL ) models described by [2], [5] and [8] do not require a choice set for model estimation. Conceptually, they are equivalent to MNL models for route choice from an infinite number of alternatives. RL uses linkadditive attributes as opposed to route attributes and conceptually applies an MNL at each junction in order to predict the next link. Hence, it can be interpreted as a link based choice model. It allows to compute the probability for a any given route.

In [2] the authors formulate a route choice model that does not rely on a prior choice set to estimate its parameters. Instead, a link-pair based choice model ( $R L$, recursive logit) is introduced. The authors prove that the model is equivalent to an MNL for an infinite number of alternatives. The deterministic utility component used to determine the probability to choose the successor link $a$ after having reached link $k$ consists of the link specific utility for $a$ and a value function term (for the Bellman algorithm), returning the expected value for the maximum utility to draw from the remaining part of the route to the destination (after choosing successor $a$ of $k$ ).

[2] also introduces the link size (LS) attribute similar to the path size attribute in PSL to correct for correlation between overlapping routes. This attribute is determined by first applying the $R L$ method using an initial estimate for the coefficients and then using the resulting link-use probability as a proxy for route overlap. The link flow proxy values need to be computed for each given OD-pair in order to evaluate the route probabilities. These proxy values indicate overlap for routes for a particular OD-pair. Solution of a system of linear equations is required for each OD-pair. As soon as the link-flow proxies are known, they can be added as an additional (link-additive) link attribute. 


\subsection{Path Complexity}

The complexity of a given path in a graph is the minimum number of BPC (Basic Path Component) in the decomposition of the path where a BPC is defined as either a least cost path or a non-least cost edge. A non-least cost edge is an edge $e$ whose vertices are connected by a bypass having a lower cost than the cost to traverse $e$. Note that a path may have multiple minimum decompositions. In this paper, distance is used as the generalized cost.

Formal definitions, the decomposition algorithm and associated mathematical proof and several frequency distributions for (mostly) car trips are presented in [4]. The majority of the trips (0.95) has complexity of at most 5.

[3] introduces the concept of Mental Representation Items (MRI) making use of a layered choice process. The first layer is used to determine a MRI choice set, such as $C_{1}=\{$ avoidCC, aroundCC, throughCC $\}$ where CC stands for city center. A layer on top of that provides additional details. In order to make the choice set operational, an attribute is assigned to each MRI by calculating the expected maximum utility and taking the sums of the logarithms of all utilities on the path. The MRI are determined interactively by the analyst but are related (although not equivalent) to the intermediate destinations in the decomposed paths.

[7] reports the complexity for $282 \mathrm{k}$ routes observed for bicyclists in The Netherlands made available in the fietstelweek2016 dataset. The observed complexity is significantly larger than the one mentioned above: 0.95 of the routes has complexity of at most 11 . This was compared to the complexity for the routes generated by POSDAP DSCSG for the observed set of OD-pairs.

\section{Dataset Properties}

Using observed GPS traces to analyze route choice behavior requires an elaborate process consisting of (i) trip identification, (ii) map matching and (iii) path decomposition. Although it is not easy to quantify in a formal way the effect of data pre-processing, all steps performed during data cleaning should be rigorously specified in order to allow data quality assessment w.r.t. particular properties. This is because particular pre-processing steps may affect results (e.g. map matching properties are crucial for path complexity analysis). Unfortunately, pre-processing details are not revealed for many publicly available datasets (e.g. the fietstelweek2016 dataset used in this research which provides routes as link sequences resulting from GPS map-matching).

\subsection{Basic Properties}

The fietstelweek2016 dataset contains $282 \mathrm{k}$ observed routes for a short survey period ( 1 week only). This is to be compared to [1] who use a 6-week period to extract systematic mobility about visited locations and chosen routes (which, according to the reported results and discussion, seems to be a minimum in order to discover behavior).

To achieve anonymization, trip start times are discretized into 1-hour buckets and person identifiers were removed. Furthermore, near the begin and end of the trip a part or random length is removed (head/tail stripping). Due to the short survey period and the anonymization (by data manipulation in both space and time) recurrent trips are missing.

Trip purpose was not recorded and it cannot be derived from repeated location visits due to head-tail stripping (see Section 3.4).

The associated GIS database considers each link to be bidirectional and it contains many trivial nodes. In GIS trivial nodes are used to specify road link geometry and to delimit segments where attribute values change.

Definition 3.1 (Trivial Node). A trivial node corresponds to a vertex having exactly two neighbours.

Definition 3.2 (String). A string in graph $G$ is a sub-graph consisting of a path in $G$ for which all vertices except the first and last ones are trivial.

In the fietstelweek2016 dataset 532851 out of 903250 (59.0\%) nodes are trivial. The presence of trivial nodes does not affect the complexity of a path but it masks recurrent use of source/destination link pairs.

\subsection{OD-Pair problem}

Each link in the dataset was mapped to its containing string and each string was represented by its head link. The use of strings instead of links mitigates the problem of low recurrent use of source/destination link-pairs. 
Table 1: The number of unique links resp. strings used as head(tail) in a route. ReUseFactor is defined a the quotient TotalNrOfRoutes/UniqueLinks resp. TotalNrOfRoutes/UniqueStrings in each row.

\begin{tabular}{lrrrr}
\hline UsedAs & UniqueLinks & ReUseFactor & UniqueStrings & \\
\hline head & 182549 & 1.549 & 165169 & 1.712 \\
tail & 189115 & 1.495 & 172081 & 1.643 \\
\hline
\end{tabular}

Table 2: OD-pair re-use based on embedding strings.

\begin{tabular}{lrrrr}
\hline Schema & nRoutes & nUniqueOD & nReUsedOD & ReUseFactor \\
\hline complete & 282795 & 276901 & 5894 & 1.021 \\
_amsterdam_c & 33178 & 29752 & 3426 & 1.115 \\
\hline
\end{tabular}

The number of unique head and tail links resp. strings in the fietstelweek2016 dataset were counted: results are summarized in Table 1. The 2-nd and 3-rd columns apply to head/tail links. The 4-th and 5-th columns apply to their respective embedding strings. For head/tail string re-use, fat tail distributions are found. A use frequency of 132 was found whereas 0.91 of the strings is re-used at most 10 times and 0.97 of the strings is re-used at most 15 times. Link and string re-use were investigated for both head and tail. Results are shown in Table 1.

$O D$-pair re-use obviously is much smaller. Embedding strings for the head and tail links were used to count unique OD-pairs. This results in Table 2 which shows that re-use is low and more than half of the re-use occurs for the _amsterdam_c facet covering the city center (see Section 4). Low ReUseFactor values are caused (i) by the short survey period (1 week) and (ii) by anonymization (see Section 3.4). As a consequence no home-work recurrent travel can be extracted for comparison with e.g. OViN (Onderzoek Verplaatsingen in Nederland) by Centraal Bureau voor de Statistiek. Finally, even $31 \mathrm{k}$ routes for Amsterdam or $282 \mathrm{k}$ routes for The Netherlands is not much because of the huge number of possible OD-pairs and routes in the respective road network graphs.

\subsection{Missing Link Problem}

Unexpectedly large string length values were found in some (rural) regions. It was observed that links are missing in the fietstelweek2016 network. Since no information about the map matching process nor the raw GPS data are available, the effect on the reported link sequences currently cannot be evaluated.

\subsection{Effects from Anonymization by Head/Tail Stripping}

The string length distribution is required to estimate the error induced by stripping parts of random length $L \sim$ $U(0,400)[\mathrm{m}]$ at the head and at the tail of each route. If the probability to strip more than one string is low (i.e. the expected stripped length is much less than the string length), then the correct string may have been identified which means that head/tail stripping does not (severely) affect the distribution for the path complexity.

The length distribution was determined for strings embedding a link that overlaps the Amsterdam bounding box because after visual inspection it can be provisionally assumed that the missing link problem (see Section 3.3) is less severe in the Amsterdam region than in other regions. Figure 1a shows the distribution (normalized CDF and PDF) for the string length measured along the road (up to $1000[\mathrm{~m}]) .29835$ of the 30453 strings $(0.9797$ ) are shorter than $400[\mathrm{~m}]$ (the value used for head/tail stripping) and $27765 / 30453(0.9117)$ are shorter than $200[\mathrm{~m}]$ (the expected value for the stripped length). Also, note that 14 522/30 453 (0.4768) of the strings have a length of at most 50[m]. Assume that head/tail stripping uses linear referencing (i.e. exactly the sampled strip length $l_{s}$ is removed from the head or tail). Let $f\left(l_{s}\right)$ denote the probability to sample the value $l_{s}$. Since $l_{s} \sim U\left(0, L_{s}\right)$, the density function $f\left(l_{s}\right)=\frac{1}{L_{s}}$. Let $F(x)$ denote the cumulative distribution for the string length (given in Figure 1a). Then the probability to remove at most one string is given by the expected value

$$
E\left[f\left(l_{s}\right) \cdot\left(1-F\left(l_{s}\right)\right)\right]=\int_{l_{s}=0}^{l_{s}=L_{s}} f\left(l_{s}\right) \cdot\left(1-F\left(l_{s}\right)\right) \partial l_{s}=\frac{1}{L_{s}} \cdot \int_{l_{s}=0}^{l_{s}=L_{s}}\left(1-F\left(l_{s}\right)\right) \partial l_{s}
$$




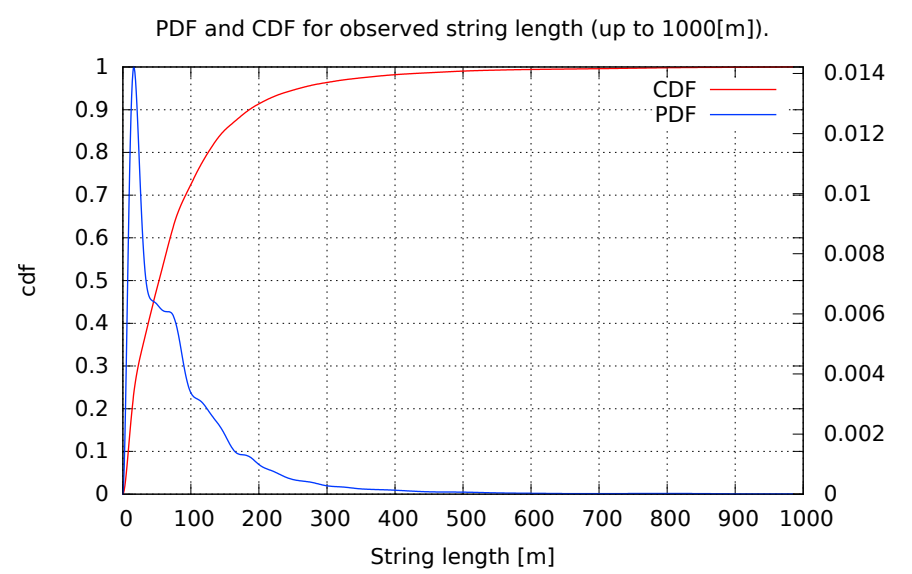

(a) Distribution for the length (up to $1000[\mathrm{~m}]$ ) of strings embedding links that overlap the Amsterdam bounding box.

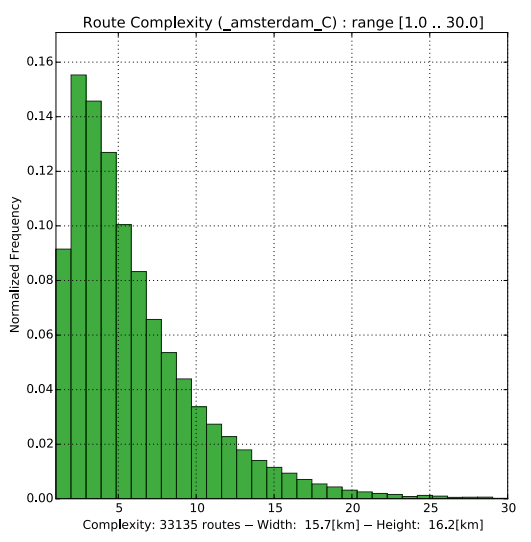

(b) Complexity PWF for Facet _amsterdam Facet $\mathrm{C}$, all trips of the day

Fig. 1: Amsterdam Central Facet: Descriptive statistics

where the factor $\left(1-F\left(l_{s}\right)\right)$ gives the probability that the head/tail string length is larger than the sampled value $l_{s}$. The number of edges in the path is the walkSize. The observed ratio walkSize/complexity (after trivial node removal) is 5 to 7 for city centers and 7 to 9 for rural areas. As a consequence, in an urban area, about 6 additional edges increase the complexity by one. Adding/deleting a single string to a given path changes the complexity by at most one. We assume that the number of strings to be added (removed) to increase (decrease) the complexity of a path obeys a Poisson distribution with $\lambda=6$. The observed PDF $\left(f_{1}(x)\right)$ shown in Figure 1a was used to compute the $f_{k}(x)$ for the sum of $k$ variables having density $f_{1}(x)$. The probability to change the path complexity by one-side stripping is given by $\bar{p}=\int_{\ell=0}^{\ell=L_{S}} f^{S}(\ell) \cdot\left(\sum_{r \in[1, \bar{r}]}\left[p_{\text {Pois }}(r \mid \lambda) \cdot \int_{l=0}^{l=\ell} f_{r}^{S L}(l) \partial l\right]\right) \partial \ell$ where $f^{S}(\ell)$ is the PDF for the sampled strip length and $f_{r}^{S L}(l)$ is the PDF for the length of $k$ concatenated strings. For $L_{S}=400$ (the value used in fietstelweek2016) $\bar{p}=0.277$ and hence the probability that both-side stripping does not affect complexity $(1-\bar{p})^{2}=0.523$

\section{Preliminary Results}

The dataset is divided into 16 regions corresponding to the main cities in The Netherlands. Each region consists of 9 facets. The central facet is the interactively defined bounding box for the city center. The other facets (labeled N,NE,E,SE,S,SW,W,NW) are defined by considering a $10[\mathrm{~km}]$ wide band on the four sides of the central one. There are three larger disjoint regions (zuiden, midden, noorden) that together cover the entire country.

\subsection{Route Length}

The distribution for the ratio $R$ between the actual route length and the shortest path length seems not to vary much. $R$ barely ever exceeds 1.4 and in many cases $R \leq 1.2$. The case for facet_amsterdam_C is shown in Figure 2a.

\subsection{Mean complexity for facets: spatialeffect}

The distribution for the complexity differs between facets. First we looked whether a pattern could be found by distinguishing urban and suburban facets (or regions). It turns out that such pattern does not occur although the probability distribution for the average complexity seems to depend on the location. Several potential factors have been investigated to predict the mean complexity found for a facet: (i) graph density in the facet (ii) number of network nodes per unit area (kind of geometric density) in the facet (iii) average length for the links in the facet (iv) ratio of 


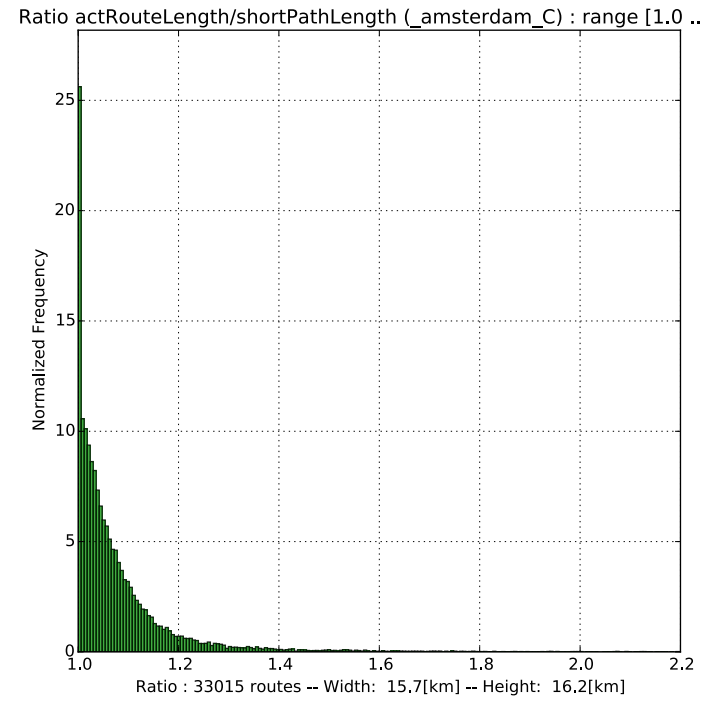

(a) Region_amsterdam Facet C, PWF for ActualLength/ShortPathLength ratio

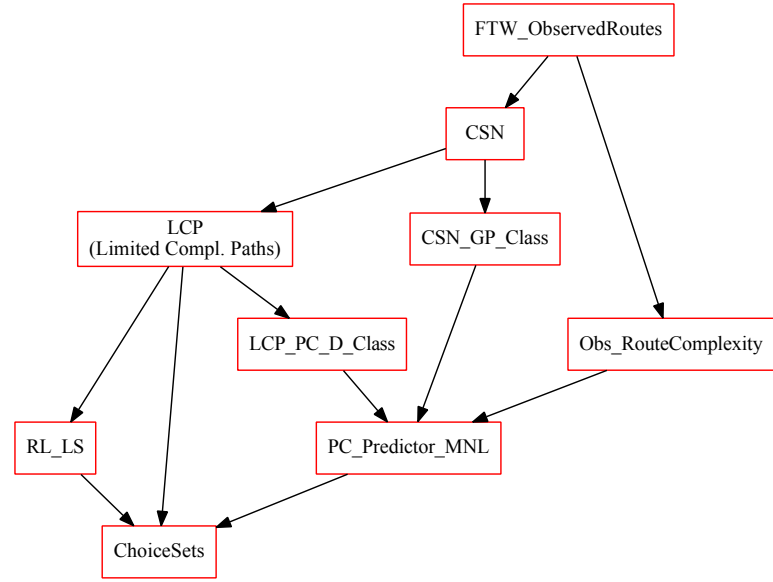

(b) Choice Set Generation: Information Flow Overview

Fig. 2: Distance ratio to construct CSN - Components Dependency

actual trip distance to shortest path length (linking $\mathrm{O}$ to $\mathrm{D}$ ). None of these seem to lead to a usable correlation. The facets may be too large, hence insufficiently specific and take irrelevant data into account.

There is a low correlation with the walk size (number of links in the route). In [6] the author shows that the correlation with the walk distance along the road is near to zero.

\subsection{Mean complexity for facets: temporal effect}

The trips for each facet were assigned to a group based on the one-hour time slot (bucket) in which the first link of the trip was crossed. The groups are morningPeak (07h-09h), offPeak (10h-15h) and eveningPeak (16h-18h). For each facet-period combination the mean and standard deviation were collected in a table. In many cases, the mean complexity for offPeak is the smallest of the three values. For each facet where the mean value for offPeak is the smallest of the three values, it is compared to the next larger one. The t-statistic is computed using

$$
t=\frac{\bar{X}_{1}-\bar{X}_{2}}{s_{p} \cdot \sqrt{\frac{1}{n_{1}}+\frac{1}{n_{2}}}} \quad s_{p}=\sqrt{\frac{\left(n_{1}-1\right) \cdot s_{X_{1}}^{2}+\left(n_{2}-1\right) \cdot s_{X_{2}}^{2}}{n_{1}+n_{2}-2}}
$$

For the aggregate regions (zuiden, midden, noorden) the mean value for the complexity in the offPeak period is significantly lower than for the other periods. This also holds for some but not all facets (e.g. $t=-6.764$ for_amsterdam_C). A possible explanation is that this is caused by city centers being crowded by pedestrians during the peak periods.

\section{Research Project Setup}

Due to the OD-pair problem it is not possible to determine distributions for the complexity of paths for particular OD-pairs in a direct way (even not after aggregating locations using $1[\mathrm{~km}]$ grid cells). Therefore, the next project stage is based on classifiers for consideration sub-networks (CSN) and limited complexity path sets (LCP). An MNL model 
is estimated using the observed routes to predict the complexity from the CSN class and LCP class. Finally, the choice set is populated by enumerating routes in the LCP and retaining the ones having a maximal likelihood based on the $R L+L S$ model and the MNL complexity predictor. Details are explained in the subsections. An overview is shown in Figure 2b.

\subsection{CSN}

For nearly all routes the ratio $\frac{\text { actLength }}{\text { shortLength }} \leq 1.4$. We define the consideration subnetwork for the pair $\langle O, D\rangle$ as the graph $G_{O, D}^{C}\left(V_{O, D}^{C}, E_{O, D}^{C}\right)$ by selecting the vertex set $V_{O, D}^{C} \subseteq V^{T}$ so that

$$
\forall v \in V_{O, D}^{C}: d(O, v)+d(v, D) \leq \alpha \cdot d_{S}(O, D)=\alpha \cdot \text { shortLength }
$$

where $d_{S}(\cdot, \cdot)$ denotes the shortest distance between two vertices and $\alpha$ follows from $\operatorname{Prob}\left(\frac{\text { actLength }}{\text { shortLength }} \leq \alpha\right)=0.99$ and $V^{T}$ is the set of road network vertices. The CSN concept is similar (but not equivalent) to the space-time prism concept which is based on travel time. Note that a digraph is used to model the road network. The CSN can be constructed from two shortest path trees: $S P T_{\text {from }}(O)$ starting at the origin and $S P T_{t o}(D)$ leading to the destination.

For estimation of RL+LS parameters the CSN shall at least contain every used link in the route. It is possible that the CSN does not contain each link in the observed route. In such cases, the distance along the road for the observed route $d_{o b s}>\alpha \cdot d_{\text {short }}(O, D)$. We opt to take the value for $\alpha$ sufficiently large and to discard the routes not meeting the threshold condition as outliers. Note that the number of edges in the CSN equals the size of the set of linear equations that needs to be solved for each OD-pair to determine the path probability in $R L+L S$ (which is a reason to keep the value for $\alpha$ small).

The aim is to build a classifier that relates the observed complexity to graph properties of the CSN (average vertex degree $\frac{\left|E_{O, D}^{C}\right|}{\left|V_{O, D}^{C}\right|}$, density $\frac{\left|E_{O, D}^{C}\right|}{\left|V_{O, D}^{C}\right| \cdot \mid\left(\left|V_{O, D}^{C}\right|-1\right)}$, the ratio $\frac{\mathcal{D}\left(G_{O, D}^{C}\right)}{|V(r)|}=\frac{|V(\bar{p})|}{|V(r)|}$ where $\mathcal{D}(\cdot)$ denotes the graph diameter and $\bar{p}$ denotes the longest shortest path in $G_{O, D}^{C}$, etc). The classifier is represented by the box labeled CSN_GP_Class in Figure 2b. By using the CSN, parts of the road network graph that are irrelevant for the trip are ignored.

\subsection{Limited Complexity Paths (LCP)}

A recursive algorithm is developed to enumerate the set of limited complexity paths (LCP) for an OD-pair: this is the set of all paths $p$ for which complexity $c(p) \leq \bar{c}$ linking $\mathrm{O}$ to $\mathrm{D}$ in $\operatorname{CSN}(\mathrm{O}, \mathrm{D})$. The value $\bar{c}$ will be chosen so that $\bar{c}$ is minimal while $F^{o b s}(\bar{c}) \geq p_{0}$ where $p_{0}$ is a given probability e.g. $p_{0}=0.99$. The algorithm will be applied to the CSN of each observed route. This delivers the PWF $f_{c}^{l c p}(O, D, \bar{c})$. The PWF's are used as follows:

1. The first aim is to find out whether the complexity of the observed route is improbable according to $f_{c}^{l c p}(O, D, \bar{c})$ (which suggests behaviour plays a significant role) or rather probable in case a random route was chosen (which means that behavioural effects are obscured). Let $c^{o b s}$ denote the complexity of the observed route linking $\mathrm{O}$ to $\mathrm{D}$. The distributions for the following values over the complete set of observations will be analyzed:

(a) the occurrence probability for $c^{o b s}$ given by $\operatorname{Prob}\left(c^{o b s}\right)=f^{l c p}(c ; O, D, \bar{c})$

(b) the value $\left|c^{o b s}-\underset{c \in[1, \bar{c}]}{\arg \max } f^{l c p}(c ; O, D, \bar{c})\right|$

2. Second, a limited set of typical complexity distributions (PWF) is to be established by clustering based on the first $k$ moments of the $f^{l c p}(c ; O, D, \bar{c})$ functions. This classifier is represented by the box labeled LCP_PC_D_Class in Figure 2b.

\subsection{Complexity Predictor}

CSN and LCP categories allow to create collections of similar routes for which the complexity distribution can be evaluated. This should solve the problem of insufficient observations for each OD-pair. An MNL model is estimated to establish a PWF for the complexity using the CSN and LCP classes as independent variables. 


\subsection{Choice Set Generation}

Assume that for an OD-pair $\langle o, d\rangle$ a choice set of $N_{P}$ paths is required in a micro-simulation for sampling. The aim is to use a subset of the LCP (see Section 5.2) as a replacement for BFS-LE and DSCSG to create the required choice set. Take into account that in some cases $|L C P|<N_{P}$ : a particular CSN may contain only a few paths that in addition are of low complexity. The path probabilities estimated by means of the RL+LS method described in [2] and the PWF for the complexity determined by the complexity predictor are combined to determine the path likelihood. Let $N=\max \left(N_{P},|L C P|\right)$; we retain the $N$ paths from LCP having the largest likelihood values. Hence, the resulting choice set takes both link attributes and observed path complexity into account.

The procedure is based on prior evidence from observations that large deviations from the shortest path are never used. For each OD-pair we assign a negative infinite link utility to the links that are certainly not used for a route connecting $\mathrm{O}$ to $\mathrm{D}$. In the RL+LS method we set $v_{a}=-\infty$ for each edge $a$ that is not considered by the traveler in a route for the given OD-pair.

This is equivalent to use the CSN to determine the route probability in the model training stage.

As a consequence, the matrix $\mathbf{M}$ in equation (7) in [2] and equation (8) in [8] has dimension $\left|E_{C S N(O, D)}\right|$ where $E_{C S N(O, D)}$ is the set of edges CSN for the pair $\langle O, D\rangle$.

Since CSN are used, the RL+LS method requires the solution of only small sets of linear equations for each observed OD-pair (much smaller than the ones used in [8]). Furthermore, the problem is embarrassingly parallel and hence computation is expected to be feasible.

\section{Conclusion}

Publicly available observed route data contain few routes for each OD-pair. A method is proposed to classify ODpairs as sufficiently similar for use in complexity prediction. A research project setup is proposed to generate choice sets for microsimulation from observed routes. A path generation technique is proposed. The likelihood for the paths that populate the choice set is derived from observations and based on link properties and on the path complexity.

\section{Acknowledgements}

The research received funding from STAR Cluster, The Netherlands.

\section{References}

[1] Bucher, D., Mangili, F., Cellina, F., Bonesana, C., Jonietz, D., Raubal, M., 2019. From location tracking to personalized eco-feedback: A framework for geographic information collection, processing and visualization to promote sustainable mobility behaviors. Travel Behaviour and Society 14, 43 - 56. URL: http://www. sciencedirect.com/science/article/pii/S2214367X18300887, doi:10.1016/j.tbs . 2018.09 .005$.

[2] Fosgerau, M., Frejinger, E., Karlstrom, A., 2013. A link based network route choice model with unrestricted choice set. Transportation Research Part B 56, 70-80. doi:10.1016/j.trb.2013.07.012.

[3] Kazagli, E., Bierlaire, M., Fltterd, G., 2016. Revisiting the route choice problem: A modeling framework based on mental representations. Journal of Choice Modelling 19, 1 -23. URL: http://www.sciencedirect.com/science/article/pii/S1755534515300518, doi:10.1016/j.jocm.2016.06.001.

[4] Knapen, L., Hartman, I.B.A., Schulz, D., Bellemans, T., Janssens, D., Wets, G., 2016. Determining structural route components from GPS traces. Transportation Research Part B: Methodological 90,156-171. URL: http://www.sciencedirect.com/science/article/pii/ S0191261516302296, doi:10.1016/j.trb.2016.04.019.

[5] Mai, T., Fosgerau, M., Frejinger, E., 2015. A nested recursive logit model for route choice analysis. Transportation Research Part B: Methodological 75, 100 - 112. URL: http://www.sciencedirect.com/science/article/pii/S0191261515000582, doi:10.1016/j.trb. 2015.03 .015$.

[6] Wardenier, N., 2017. On Bicycle Choice Set Generation. Master's thesis. UUtrecht. Utrecht, The Netherlands. URL: https://dspace. library.uu.nl/handle/1874/355825.

[7] Wardenier, N., Knapen, L., Koch, T., Dugundji, E., 2019. Improving bicycle route choice set generation using route complexity in GPS traces, in: TRB 2019 Annual Meeting, Transportation Research Board, Washington, D.C.

[8] Zimmermann, M., Mai, T., Frejinger, E., 2017. Bike route choice modeling using GPS data without choice sets of paths. Transportation Research Part C: Emerging Technologies 75, 183 - 196. URL: http://www.sciencedirect.com/science/article/pii/ S0968090X16302637, doi:10.1016/j.trc.2016.12.009. 